\title{
ConFIRM trial - conversion of in vitro fertilization cycles to intrauterine inseminations in patients with a poor ovarian response to stimulation: a protocol for a multicentric, prospective randomized trial
}

Léa Delbos ${ }^{1 *}$ D, Elsa Parot-Schinkel ${ }^{2}$, Hady El Hachem ${ }^{3}$, Guillaume Legendre ${ }^{1}$, Philippe Descamps ${ }^{1}$, Lisa Boucret ${ }^{1}$, Véronique Ferré-L'Hotellier ${ }^{1}$, Pauline Jeanneteau' ${ }^{1}$, Cécile Dreux ${ }^{1}$, Catherine Morinière ${ }^{4}$, Pascale May-Panloup ${ }^{1}$ and Pierre-Emmanuel Bouet ${ }^{1}$

\begin{abstract}
Background: To date, there is no consensus on the ideal management strategy of patients with poor ovarian response (POR) to controlled ovarian stimulation (COS) for in vitro fertilization (IVF). Currently, these patients are given the choice of: (1) canceling the cycle; (2) proceeding with COS regardless of the poor response, and performing the oocyte retrieval and transfer of embryos when available; or (3) conversion to an intrauterine insemination (IUI). When the decision to proceed with the COS cycle is taken, it is not clear whether IVF or conversion to IUI is the best choice. If live birth rates were comparable between the two strategies, conversion to IUI would be the better option for poor responders, since it is less invasive and is associated with a lower cost.
\end{abstract}

Methods: We designed a non-inferiority, multicentric, randomized controlled trial that will be conducted in 18 French Reproductive Medicine centers. We defined POR as the presence of only two or four mature follicles $\geq$ $14 \mathrm{~mm}$ on ovulation trigger day. Patients with POR will be randomized into two parallel arms: "IVF" and "conversion to IUI." Our main objective is to compare the efficiency of IVF and conversion to IUI in patients with POR to COS. The primary outcome is the live birth rate, defined as the birth of a living infant after 22 weeks' gestational age, or weighing $\geq 500 \mathrm{~g}$. One of the secondary objectives is to compare the cost-efficiency of both strategies at 12 months. We will need to include 940 patients (470 in each arm), and the duration of the inclusion period is estimated to be 36 months.

Discussion: This is the first randomized controlled trial to compare the outcomes of IVF and embryo transfer to conversion to IUI in patients with POR to COS. If our study shows that conversion to IUI is non-inferior to IVF in terms of clinical efficiency and live birth rate, it would confirm IUI as a better alternative for patients, both individually (less invasive and more patient-friendly) and collectively (lower cost).

Trials registration: ClinicalTrials.gov, ID: NCT03362489. Registered on January 10th, 2018.

Keywords: IVF, Conversion to IUI, Poor ovarian response

\footnotetext{
* Correspondence: leamtc@hotmail.fr

${ }^{1}$ Department of Reproductive Medicine, Angers University Hospital, 4 rue

Larrey, 49000 Angers, France

Full list of author information is available at the end of the article
}

(c) The Author(s). 2018 Open Access This article is distributed under the terms of the Creative Commons Attribution 4.0 International License (http://creativecommons.org/licenses/by/4.0/), which permits unrestricted use, distribution, and reproduction in any medium, provided you give appropriate credit to the original author(s) and the source, provide a link to the Creative Commons license, and indicate if changes were made. The Creative Commons Public Domain Dedication waiver (http://creativecommons.org/publicdomain/zero/1.0/) applies to the data made available in this article, unless otherwise stated. 


\section{Background}

In vitro fertilization (IVF), with or without intracytoplasmic spermatozoid injection (ICSI), followed by embryo transfer, is commonly offered for millions of couples with infertility worldwide. It is usually preceded by controlled ovarian stimulation (COS) with exogenous gonadotropins, which allows to increase the number of follicles and mature oocytes available, thus increasing the number of good-quality embryos available for transfer, and improving the pregnancy and live birth rates (LBRs). However, in about $6 \%$ of COS cycles for IVF, less than five mature follicles are obtained despite the use of high doses of exogenous gonadotropins $[1,2]$. These cases are referred to as "poor ovarian response to stimulation" (POR), and are associated with lower pregnancy and LBRs. When managing patients with POR during a COS cycle, there are three available options. The first is to proceed with the stimulation despite the poor response, and perform the oocyte retrieval and transfer the available embryos. However, there is a high risk of failure to retrieve oocytes and the absence of embryos to transfer, which should be weighed against the risks of complications associated with the oocyte retrieval and anesthesia. Moreover, such events could be the source of significant disappointment and stress for the couple, and they should be thoroughly counseled before proceeding with the stimulation $[3,4]$. The second option is conversion of the IVF cycle to an intrauterine insemination (IUI) in patients having at least one patent tube and adequate semen parameters $[5,6]$. Conversion to IUI allows the couple to benefit from the treatments already administered, without the risk of an oocyte retrieval/embryo transfer failure. Finally, the third option is to cancel the IVF cycle. The decision to cancel a cycle is always a challenging one, since it involves not only clinical, but also emotional and financial considerations. Indeed, it is often difficult to announce to a couple the decision to abandon a COS cycle following several weeks of preparation, and after the patient has had several days of high-dose gonadotropin injections. Moreover, several studies have shown that in women with POR, it remains advantageous to proceed with the planned assisted reproductive treatment (ART) [7].

In 2011, the European Society of Human Reproduction and Embryology (ESHRE) published the Bologna criteria in order to "standardize the definition of POR in a simple and reproducible manner" [3, 4]. At least two of the following three criteria are required to establish the definition of POR: (1) advanced maternal age ( $>40$ years) or any other risk factor for POR; (2) a previous POR (at least three oocytes with a conventional stimulation protocol); (3) an abnormal ovarian reserve test (i.e., antral follicle count (AFC) less than five to seven follicles or anti-Müllerian hormone (AMH) below 0.5-1.1 ng/
$\mathrm{mL}$ ). Moreover, two episodes of POR after maximal stimulation are enough to define a patient as a poor responder according to the Bologna criteria, in the absence of advanced maternal age or abnormal ovarian reserve [3]. However, ever since their publication, these criteria have been the subject of many debates and several revisions have been suggested and requested [5]. Indeed, some aspects have been criticized, such as the supposed homogeneity of the population, the defined thresholds for age, AFC and AMH, the proposed risk factors other than age, and the lack of consideration for oocyte quality compared to quantity [5].

Only five studies compared the outcome of IVF and conversion to IUI in patients with POR [2, 8-11]. These studies have reported that there is no benefit in proceeding with IVF in the presence of only one mature follicle. However, the results in cases with two, three or four mature follicles were divergent. Indeed, three studies reported the superiority of IVF $[2,8,9]$, whereas the two others failed to show a difference between IVF and conversion to IUI $[10,11]$. Unfortunately, these studies have low levels of evidence, mostly because of their retrospective observational designs, and the presence of multiple biases [2, 8-11]. Therefore, the ideal management of patients with two, three or four mature follicles following COS remains unknown [12]. This is why we have decided to perform the first randomized controlled trial (RCT) to compare these two treatment methods. If our study shows that conversion to IUI is non-inferior to IVF in terms of clinical efficiency and LBR, it would confirm IUI as a better alternative for patients, both individually (less invasive and more patient-friendly) and collectively (lower cost).

\section{Methods}

\section{Aims and outcome measures}

The primary objective is to compare the efficiency of IVF and conversion to IUI in patients with a poor ovarian response to COS. The main outcome measure is the LBR, defined as the birth of a living infant after 22 weeks' gestational age (GA), or weighing $\geq 500 \mathrm{~g}$.

The secondary objectives are: (1) to compare the outcomes of IVF and conversion to IUI in women with POR to COS: biochemical pregnancy rate, clinical pregnancy rate, pregnancy loss rate, multiple pregnancy rate, mean term at delivery and postnatal outcomes; (2) to compare the impacts of IVF and conversion to IUI on overall outcomes in women with POR to COS, according to the number of mature follicles on trigger day (2 vs 3 vs 4 ), and according to age ( $<40$ years vs $\geq 40$ years); (3) to compare the impacts of IVF and conversion to IUI on the cumulative clinical pregnancy and LBRs - taking into account frozen embryo transfers in IVF - in women with POR to COS; (4) to compare the clinical efficiency 
of IVF and conversion to IUI in women considered "poor ovarian responders" according to the Bologna criteria; (5) to analyze the rate of IVF cycles with failed oocyte retrievals (no oocytes) and no embryo transfers and (6) to compare the cost-efficiency of both strategies at 12 months.

The secondary outcomes measures are:

- Biochemical pregnancy rate: defined as serum human chorionic gonadotropin (HCG) levels > $10 \mathrm{IU} / \mathrm{L}, 14$ days after the IUI or the embryo transfer, followed by a rapid decrease until being undetectable

- Clinical pregnancy rate: defined as fetal cardiac activity at 6-7 weeks' GA

- Spontaneous pregnancy loss (PL) rate: including early and late pregnancy losses

- Multiple pregnancy rate: defined as more than two embryos visualized on ultrasound at 7 weeks' GA

- Term at delivery, neonatal complications and survival

- All outcome measures will be further analyzed according to patients' age ( $<40$ years vs $\geq 40$ years) and the number of follicles on trigger day ( 2 vs 3 vs 4 )

- All outcome measures will be further analyzed in the subgroup of women considered poor responders according to the Bologna criteria

- The rate of IVF cycles with failed oocyte retrievals and no embryo transfers

- Cumulative clinical pregnancy and LBRs in the IVF group, taking into account fresh and frozen embryos transferred in subsequent cycles

- Cost-efficiency analysis at 12 months

\section{Trial design}

This is a prospective, multicenter, parallel-group, open-label randomized controlled and non-inferiority trial conducted at 18 French departments of reproductive medicine (Table 1).

The study protocol was designed using the recommendations of the Consolidated Standards of Reporting Trial (CONSORT) Statement, Recommendations for Interventional Trials (SPIRIT) 2013 Checklist (Additional file 1) and according to the guidelines of cost-effectiveness studies of the French Health Authority. Eligible patients will be randomized equally to either conversion to IUI or IVF.

\section{Recruitment}

Patients will be recruited in all participating reproductive medicine centers across France. Potentially eligible patients will be pre-identified by the investigator or the co-investigators, based on their medical files and the different selection criteria that can be evaluated at the time, without informing these patients. Patients will be identified during ultrasound monitoring of follicular growth in the course of COS, and those with POR will be handed an information letter explaining the study protocol, so that they are already fully informed by the time they reach ovulation trigger day. The inclusion visit will take place on the day the decision to trigger ovulation is taken. During the inclusion visit, the investigator will thoroughly explain the study and hand the patient an information letter, written in an easily accessible language. The patient who wishes to be enrolled will sign the consent form, and will be randomized to one of the two groups. Randomization will be performed centrally by the trial coordinators using an online randomization module (Ennov $\mathrm{Clinical}^{\circ}$ ) in a 1:1 allocation ratio between IVF and conversion to IUI, as shown in Fig. 1. Moreover, randomization will be dynamic, and stratified by: center, number of mature follicles (two, three or four) and age ( $<40$ or $\geq 40$ years).

\section{Eligibility criteria}

The inclusion criteria are: (1) patients who accepted being included and signed the consent forms; (2) age $\geq 18$ and $<43$ years and (3) IVF cycle, with and without ICSI, using the "conventional" agonist (long and short) or antagonist protocol, using urinary or recombinant gonadotropins, and having only two, three or four mature follicles $(\geq 14 \mathrm{~mm})$ on the ovulation trigger day.

Table 1 French partners of study

\begin{tabular}{ll}
\hline Gynecologists & Center/department \\
\hline $\begin{array}{l}\text { Dr Pierre-Emmanuel } \\
\text { Bouet }\end{array}$ & $\begin{array}{l}\text { Coordinating investigator, Angers University Hospital, } \\
\text { Dr Florence Leperlier }\end{array}$ \\
Dr Claire de Vienne & Nantes University Hospital, Nantes \\
Dr Nathalie Massin & Créteil University Hospital, Créteil \\
Dr Mathilde Domin- & Rennes University Hospital, Rennes \\
Bernhard & \\
Dr Marion Cornuau & Tours University Hospital, Tours \\
Dr Olivier Pirrello & Strasbourg University Hospital, Strasbourg \\
Dr Catherine Morinière & Pointe-à-Pitre University Hospital, Guadeloupe \\
Dr Sophie Fressard & Lorient Public Hospital, Lorient \\
Dr Marie-Laure Langlois & Clinique Jules Verne, Nantes \\
Dr Anne-Cécile Racine & Polyclinique de l'Atlantique, Saint Herblain \\
Dr Anne Guivarc'h- & Clinique de la Sagesse, Rennes \\
Levêque & \\
Dr Frédéric Lamazou & Clinique Pierre Cherrest, Neuilly-sur-Seine \\
Dr Xenia Lechat & Polyclinique Jean Vilar, Bruges \\
Dr Claudine Vasseur & Clinique Léonard de Vinci, Chambray-lès-Tours \\
Dr Aurore Guennifey & Grenoble University Hospital, Grenoble \\
Pr Blandine Courbière & Marseille University Hospital, Marseille \\
Pr Michaël Grynberg & Antoine Béclère Hospital, Clamart \\
\hline & \\
&
\end{tabular}


The exclusion criteria are: (1) confirmed bilateral tubal occlusion; (2) non-French speaking patients; (3) partners with severe oligoasthenoteratospermia (OATS) (less than five million motile spermatozoa in the ejaculate); (4) suboptimal stimulation protocols: protocols $\leq 150$ IU of daily gonadotropins or mild stimulation protocols or natural and modified natural cycle protocols; (5) couples undergoing IVF for Preimplantation Genetic Diagnosis (PGS) or Preimplantation Genetic Screening (PGS); (6) women under legal guardianship; (7) women with no health or social security coverage and (8) women participating in other interventional trials.

\section{Study population}

At our center, in 2014, we performed 30 IVF cycles following COS with high doses of gonadotropins ( $\geq 300 \mathrm{IU}$ ) in patients at risk of POR. Our clinical pregnancy rate (CPR) was $10 \%(3 / 30)$, and our LBR was $10 \%$ $(3 / 30)$. During that same year, we had 37 IVF cycles which were converted to IUI because of POR. The CPR was $11 \%(4 / 37)$ and $L B R$ was $5 \%(2 / 37)$. If we consider that a maximal difference of $5 \%$ between IVF and conversion to IUI is clinically acceptable in order to consider conversion to IUI to be non-inferior to IVF, we will need to include 940 cycles, 470 in each group, to confirm the non-inferiority, with a power of $80 \%$, an $\alpha$ risk of $5 \%$ and with $5 \%$ of non-evaluable cycles. It is worth noting that patients who are excluded from the study for failure of treatment can be included later on during their next IVF cycle, considering they still fulfill the inclusion criteria.

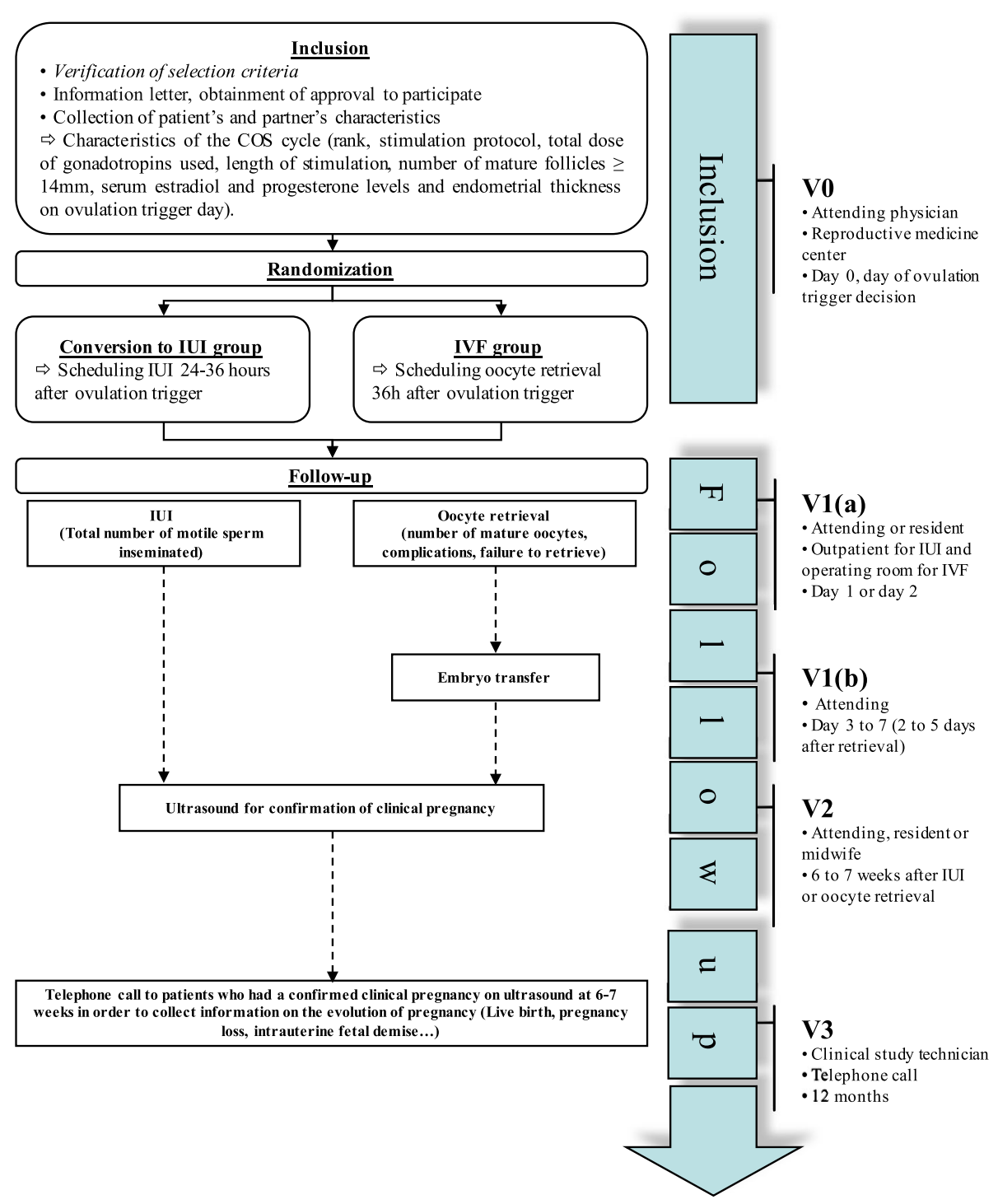

Fig. 1 Flow chart. COS controlled ovarian stimulation, $V$ visit, IUI intrauterine insemination, IVF in vitro fertilization 


\section{Procedures}

Patients will be recruited on the day of ovulation trigger. Ovulation will be triggered with an injection of urinary HCG (administered intramuscularly or subcutaneously, 5000 or 10,000 units), or recombinant HCG, depending on the participating center's protocol.

Patients will be randomized into two parallel arms (Fig. 2):

- In the "IVF" arm, oocyte retrieval is performed $36 \mathrm{~h}$ after the HCG injection, in the operating room, under transvaginal ultrasound guidance, under local or general anesthesia. The procedure lasts about $20 \mathrm{~min}$ and the patients are discharged on the same day. The oocytes retrieved from the follicles are transported immediately to the laboratory for fertilization with the partner's sperm. Fertilization is done either via conventional IVF, or via ICSI, depending on the indication. Embryos are later transferred into the uterus on day 3 or day 5 , under ultrasound guidance, in the outpatient department

- In the "conversion to IUI" arm (intervention group), IUI is performed 24 to $36 \mathrm{~h}$ after ovulation trigger. The partner provides the sperm on site and on the morning of the insemination, and it will be prepared with a two-layer density gradient. Attending physicians, fellows or residents will perform the insemination in the outpatient department, using a soft catheter, with the patient lying in the gynecologic position

\section{Cost-effectiveness analysis}

One of our secondary objective is to perform a cost-effectiveness analysis, in order to determine the optimal treatment strategy for patients with POR to COS, not only in terms of efficiency, but also in terms of cost. We will compare the reference strategy (IVF) to the experimental strategy (conversion to IUI). We will use the incremental cost-effectiveness ratio (ICER), which represents the average incremental cost associated with one additional unit of the measure of effect. IECR is calculated as follows: IECR $=\Delta$ cost $/ \Delta$ effectiveness. Costs will include the pharmacological compounds, the IVF and IUI procedures, the hospitalization and the potential complications, and will be expressed in euros $(€)$. Moreover, the loss of productivity sustained by the women and their partners during the treatment will also be included in the costs. We will not include the hospital resources used for the COS monitoring (ultrasound scans, blood tests...), nor the ones for the pregnancy follow-up since the treatment method has no impact on the evolution of pregnancy once a clinical pregnancy is confirmed. We will perform a univariate sensitivity analysis (Tornado diagram) to assess the effects of several parameters on the overall outcome.
Data collection and follow-up procedures

All data will be recorded in an electronic Case Report Form (eCRF) specifically elaborated for the study (eCRF CleanWEB, Telemedicine Technologies S.A.S) and will be collected at four specific moments: upon randomization (V0), when performing the procedure (IVF or IUI) (V1), after the ultrasound at 6-7 weeks' GA to confirm the clinical pregnancy (V2) and after a telephone call 12 months later for follow-up of women who had a clinical pregnancy confirmed at 6-7 weeks (V3). In the IVF group, the procedure (V1) will be divided into: V1a: day of oocyte retrieval, and V1b: day of embryo transfer. In the conversion to IUI group, data collection will be on the day of insemination (Fig. 1).

\section{Statistical analysis}

We will perform a descriptive analysis of the population's characteristics. Categorical variables will be expressed as numbers with percentages, and compared using Pearson's chi-square test or Fisher's exact test. Continuous variables will be reported as mean values and standard deviations, or medians with 25th and 75th percentile, and compared using Student's $t$ test or Mann-Whitney's non-parametric test. All statistical tests will be bilateral and a $p$ value $<0.05$ will be considered statistically significant. We will perform an intention-to-treat and a per-protocol analysis of all outcomes. For the primary outcome, conversion to IUI will be considered non-inferior to IVF if the upper bound of the one-sided $95 \%$ confidence interval of the difference in LBRs between the two arms (the rate in IVF minus the rate in conversion to IUI) is $\leq 5 \%$. Analysis of the primary and secondary outcomes will be adjusted according to the stratification variables used for randomization. The primary outcome will be tested using the raw difference in LBRs, and no complex model incorporating the stratification variables will be used. The dynamic randomization on these variables will ensure the variables are well-balanced. Subgroup analysis will be performed according to patients' age $(<40$ vs $\geq 40$ years), the number of mature follicles (two vs three vs four) and patients considered poor ovarian responders according to the Bologna criteria. On the other hand, the cost-effectiveness analysis will be performed on an intention-to-treat basis (the hypothesis being that conversion to IUI is superior to IVF).

\section{Data circulation}

All data from the trial will be compiled in an eCRF. Only people involved in the trial will have access to the data, via a username and a password. No patient identifying information will be stored, and only the first letter of the surname and of the last name will be kept, without any mention of the full name or date of birth. Patient code 


\begin{tabular}{|c|c|c|c|c|c|c|c|c|c|}
\hline \multirow[b]{3}{*}{ TIMEPOINT } & \multicolumn{9}{|c|}{ STUDY PERIOD } \\
\hline & \multirow{2}{*}{$\begin{array}{c}\begin{array}{c}\text { Enrol } \\
\text { ment }\end{array} \\
-t_{1}\end{array}$} & \multirow{2}{*}{$\begin{array}{c}\begin{array}{c}\text { Allocat } \\
\text { ion }\end{array} \\
0\end{array}$} & \multicolumn{6}{|c|}{ Post-allocation } & \multirow{2}{*}{$\begin{array}{c}\begin{array}{c}\text { Close } \\
\text {-out }\end{array} \\
t_{x}\end{array}$} \\
\hline & & & $t_{1}$ & $t_{2}$ & $t_{3}$ & $t_{4}$ & $t_{5}$ & $t_{6}$ & \\
\hline \multicolumn{10}{|l|}{ ENROLMENT: } \\
\hline \multirow{3}{*}{$\begin{array}{r}\text { Informed consent } \\
\text { Characteristics of the cos } \\
\text { cycle }\end{array}$} & $X$ & & & & & & & & \\
\hline & $x$ & & & & & & & & \\
\hline & $X$ & & & & & & & & \\
\hline Allocation & & $\mathrm{X}$ & & & & & & & \\
\hline \multicolumn{10}{|l|}{ INTERVENTIONS: } \\
\hline \multicolumn{10}{|l|}{ [IVF] } \\
\hline [IUI] & & & $x$ & & $\mathrm{X}$ & & & & \\
\hline \multirow[t]{2}{*}{ ASSESSMENTS: } & $\mathrm{X}$ & & & & & & & & \\
\hline & & $\mathrm{X}$ & & & & & & $\mathrm{X}$ & $x$ \\
\hline $\begin{array}{r}\text { [Biochemical } \\
\text { pregnancy rate] }\end{array}$ & & & & & & $\mathrm{X}$ & & & $X$ \\
\hline $\begin{array}{r}\text { [Clinical pregnancy } \\
\text { rate] }\end{array}$ & & & & & & & $X$ & & $\mathrm{X}$ \\
\hline $\begin{array}{r}\text { [Spontaneous pregnancy } \\
\text { loss }(P L) \text { rate] }\end{array}$ & & & & & & & $X$ & & $X$ \\
\hline $\begin{array}{r}\text { [Multiple pregnancy } \\
\text { rate] }\end{array}$ & & & & & & & $X$ & & $X$ \\
\hline $\begin{array}{r}\text { [Term at delivery, } \\
\text { neonatal complications } \\
\text { and survival] }\end{array}$ & & & & & & & & $\mathrm{X}$ & $\mathrm{X}$ \\
\hline $\begin{array}{r}\text { [age (<40 years vs } \geq 40 \\
\text { years) and the number } \\
\text { of follicles on trigger } \\
\text { day (2 vs } 3 \text { vs } 4) \text { ] }\end{array}$ & $\mathrm{X}$ & $\mathrm{X}$ & & & & $\mathrm{X}$ & $X$ & $\mathrm{x}$ & $\mathrm{X}$ \\
\hline $\begin{array}{l}\text { [women considered } \\
\text { poor responders } \\
\text { according to the } \\
\text { Bologna criteria] }\end{array}$ & $\mathrm{X}$ & $\mathrm{X}$ & & & & $\mathrm{X}$ & $X$ & $\mathrm{X}$ & $\mathrm{X}$ \\
\hline $\begin{array}{l}\text { [The rate of IVF cycles } \\
\text { with failed oocyte } \\
\text { retrievals and no } \\
\text { embryo transfers] }\end{array}$ & & & $X$ & $X$ & $\mathrm{x}$ & & & & $x$ \\
\hline $\begin{array}{r}\text { [Cumulative clinical } \\
\text { pregnancy and live } \\
\text { birth rates in the IVF } \\
\text { group, taking into } \\
\text { account fresh and } \\
\text { frozen embryos } \\
\text { transferred in } \\
\text { subsequent cycles] }\end{array}$ & & & & & & $\mathrm{X}$ & $X$ & $x$ & $\mathrm{X}$ \\
\hline $\begin{array}{r}\text { [Cost-efficiency } \\
\text { analysis] }\end{array}$ & & & & & & & & $x$ & $X$ \\
\hline
\end{tabular}

Fig. 2 Standard Protocol Items: Recommendations for Interventional Trials (SPIRIT) Figure of ConFIRM study protocol

will be composed of the participating center's number and another number assigned by that center. Each participating center will store its own data for the duration set by regulation for this type of study under the center's investigator's responsibility. Only investigators from Angers University Hospital (AUH) will have access to 
patient data from other centers on the eCRF. Therefore, for centers that cannot complete patient follow-up at 12 months, patients' coordinates will be retrieved from the eCRF by AUH investigators and personnel in charge of patient follow-up. At the end of the trial, all data will be deleted. Approval from the CCTIRS (Comité consultatif sur le traitement de l'information en matière de recherche) (Advisory Committee on Treatment of Information in Research in the Field of Healthcare) and the CNIL (Commission nationale informatique et liberté) (National Commission on Informatics and Liberty) have been requested.

\section{Monitoring and adverse events}

We do not expect to have undesirable or serious undesirable events related to the study and its proceedings. Indeed, all procedures included in this study (COS and its monitoring, ovulation trigger, oocyte retrieval, embryo transfer, IUI) are standard procedures and will be performed in accordance with the national guidelines and recommendations. The only part that is specific to our RCT, and that is not considered part of routine practice, is the telephone call that will be made to patients 12 months after enrollment in order to collect information on the evolution of pregnancy. This might be a sensitive topic, especially for couples who did not have a healthy live birth because of pregnancy loss or complications of pregnancy. The personnel in charge of this part will be trained in how to approach the couples and how to deal with the special situations. Moreover, we will have a psychologist available for couples to consult when needed.

All adverse events related to the oocyte retrieval (bleeding, hemoperitoneum, infection, peritonitis, pelvic abscess, etc.) and the anesthesia during the retrieval will be recorded. We will also document whether the complications required any additional treatment, procedure or hospitalization, and add them to the treatment cost.

\section{Expected repercussions}

If LBRs were to be comparable between IVF and conversion to IUI in patients with POR to COS, and if our cost-effectiveness analysis confirms that IUI is indeed more cost-effective, then we would confirm that conversion to IUI is the better treatment option, since it is also less invasive.

\section{Discussion}

This is the first RCT to compare the outcomes of IVF and embryo transfer to conversion to IUI in patients with poor ovarian response (POR) to controlled ovarian stimulation (COS). If our study shows that conversion to IUI is non-inferior to IVF in terms of clinical efficiency and LBR, it would confirm IUI as a better alternative for patients, both individually (less invasive and more patient-friendly) and collectively (lower cost).

\section{Trial status}

The trial already began on 10 January 2018, and participant recruitment and follow-up will continue over a 48-month period, with the anticipated final follow-up telephone call(s) occurring in January 2022. Primary analyses will be complete by June 2022 .

\section{Additional file}

Additional file 1: Standard Protocol Items: Recommendations for Interventional Trials (SPIRIT) 2013 Checklist: recommended items to address in a clinical trial protocol and related documents. (DOCX $74 \mathrm{~kb}$ )

\section{Abbreviations}

AFC: Antral follicle count; AMH: Anti-Müllerian hormone; ART: Assisted reproductive treatment; AUH: Angers University Hospital; CCTIRS: Comité consultatif sur le traitement de l'information en matière de recherché = Advisory Committee on Treatment of Information in Research in the Field of Healthcare; CNIL: Commission nationale informatique et liberté = National Commission on Informatics and Liberty; CONSORT: Consolidated Standards of Reporting Trial; COS: Controlled ovarian stimulation; CPR: Clinical pregnancy rate; eCRF: Electronic Case Report Form; ESHRE: European Society of Human Reproduction and Embryology; GA: Gestational age; HCG: Human chorionic gonadotropin; ICER: Incremental cost-effectiveness ratio;

ICSI: Intracytoplasmic spermatozoid injection; IUI: Intrauterine insemination; IVF: In vitro fertilization; LBR: Live birth rate;

OATS: Oligoasthenoteratospermia; PL: Pregnancy loss; POR: Poor ovarian response; RCT: Randomized controlled tria

\section{Funding}

The research project is funded by le Programme Hospitalier de Recherche Clinique Interrégional du Grand Ouest de 2016 n49RC17_0027/ LOI API16/ $\mathrm{A} / 042$.

\section{Availability of data and materials}

The datasets generated and/or analyzed during the current study are available from the corresponding author on reasonable request.

\section{Authors' contributions}

PEB, EPS, LD, CM and PMP conceived and designed, included the power calculation. PEB and EPS acquired legal authorizations. The study protocol and manuscript have been drafted by HEH, GL, PJ, PD, LB, VFLH and CD. All authors read and approve the final manuscript.

\section{Ethics approval and consent to participate}

The Ethics Committee of Lyon South-East II (registration number: 2017-23-2) has granted ethics approval for this study. In the event of additional studies performing subanalyses based on our database, all the investigators should keep the results confidential until these are publicly available, and they cannot publish any data related to the database without the approval of the principle investigator. During the inclusion visit, the investigator will thoroughly explain the study and hand the patient an information letter, written in an easily accessible language. The patient who wishes to be enrolled will sign the consent form, and will be randomized to one of the two groups.

\section{Consent for publication}

Not applicable.

\section{Competing interests}

The authors declare that they have no competing interests. 


\section{Publisher's Note}

Springer Nature remains neutral with regard to jurisdictional claims in published maps and institutional affiliations.

\section{Author details}

'Department of Reproductive Medicine, Angers University Hospital, 4 rue Larrey, 49000 Angers, France. ${ }^{2}$ Clinical Research Unit, Angers University Hospital, Angers, France. ${ }^{3}$ Department of Reproductive Medicine, Clemenceau Medical Center, Beirut, Lebanon. ${ }^{4}$ Department of Reproductive Medicine, Pointe-à-Pitre University Hospital, Guadeloupe, France.

Received: 29 April 2018 Accepted: 25 September 2018

Published online: 17 October 2018

\section{References}

1. Abusheikha N, Lass A, Burnley A, et al. In vitro fertilization cycles converted to intrauterine insemination because of poor follicular response have low success rates. Fertil Steril. 2001;75(3):634-5.

2. Reichman DE, Gunnala V, Meyer $L$, et al. In vitro fertilization versus conversion to intrauterine insemination in the setting of three or fewer follicles: how should patients proceed when follicular response falls short of expectation? Fertil Steril. 2013;100(1):94-9.

3. Ferraretti AP, Marca AL, Fauser BCJM, et al. ESHRE consensus on the definition of "poor response" to ovarian stimulation for in vitro fertilization: the Bologna criteria. Hum Reprod. 2011;26(7):1616-24.

4. Younis JS, Ben-Ami M, Ben-Shlomo I. The Bologna criteria for poor ovarian response: a contemporary critical appraisal. J Ovarian Res. 2015;8:76.

5. Badawy A, Wageah A, El Gharib M, et al. Prediction and diagnosis of poor ovarian response: the dilemma. J Reprod Infertil. 2011;12:241-8.

6. Ardenti R, Campari C, Agazzi L, et al. Anxiety and perceptive functioning of infertile women during in-vitro fertilization: exploratory survey of an Italian sample. Hum Reprod Oxf Engl. 1999;14(12):3126-32.

7. Norian JM, Levens ED, Richter KS, et al. Conversion from assisted reproductive technology to intrauterine insemination in low responders: Is it advantageous? Fertil Steril. 2010;94(6):2073-7.

8. Quinquin $\mathrm{M}$, Mialon $\mathrm{O}$, Isnard $\mathrm{V}$, et al. In vitro fertilization versus conversion to intrauterine insemination in Bologna-criteria poor responders: how to decide which option? Fertil Steril. 2014;102(6):1596-601.

9. Nicopoullos JDM, Abdalla H. Poor response cycles: when should we cancel? Comparison of outcome between egg collection, intrauterine insemination conversion, and follow-up cycles after abandonment. Fertil Steril. 2011;95(1):68-71.

10. Shahine LK, Lathi RB, Baker VL. Oocyte retrieval versus conversion to intrauterine insemination in patients with poor response to gonadotropin therapy. Fertil Steril. 2009;92(4):1315-7.

11. Freour T, Dubourdieu S, Mirallie S, et al. IVF conversion to IUI in poor responders: an observational study. Arch Gynecol Obstet. 2010;282(4):445-9.

12. Bouet $P E$, Legendre $G$, Delbos $L$, et al. IVF versus conversion to IUI in patients with poor ovarian response to controlled ovarian stimulation. Gynecol Obstet Fertil Senol. 2018;46(2):118-23.

Ready to submit your research? Choose BMC and benefit from:

- fast, convenient online submission

- thorough peer review by experienced researchers in your field

- rapid publication on acceptance

- support for research data, including large and complex data types

- gold Open Access which fosters wider collaboration and increased citations

- maximum visibility for your research: over $100 \mathrm{M}$ website views per year

At $\mathrm{BMC}$, research is always in progress.

Learn more biomedcentral.com/submissions 